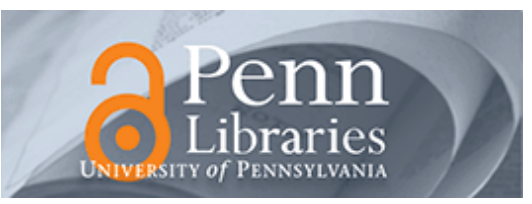

University of Pennsylvania ScholarlyCommons

September 2004

\title{
Resuscitating the Physician-Patient Relationship: Emergency Department Communication in an Academic Medical Center
}

\author{
Karin V. Rhodes \\ Teri Vieth \\ University of Chicago \\ Theresa $\mathrm{He}$ \\ University of Chicago \\ Annette Miller \\ University of Chicago \\ David S. Howes \\ University of Chicago
}

University of Pennsylvania, kvr@sp2.upenn.edu

See next page for additional authors

Follow this and additional works at: https://repository.upenn.edu/spp_papers

\section{Recommended Citation}

Rhodes, K. V., Vieth, T., He, T., Miller, A., Howes, D. S., Bailey, O., Walter, J., Frankel, R., \& Levinson, W. (2004). Resuscitating the Physician-Patient Relationship: Emergency Department Communication in an Academic Medical Center. Retrieved from https://repository.upenn.edu/spp_papers/108

Postprint version. Published in Annals of Emergency Medicine, Volume 44, Issue 3, September 2004, pages 262-267.

Publisher URL: http://dx.doi.org/10.1016/j.annemergmed.2004.02.035

At the time of publication, author Karin V. Rhodes was affiliated with the University of Chicago. Currently, she is a faculty member of the School of Social Policy and Practice at the University of Pennsylvania.

This paper is posted at ScholarlyCommons. https://repository.upenn.edu/spp_papers/108

For more information, please contact repository@pobox.upenn.edu. 


\title{
Resuscitating the Physician-Patient Relationship: Emergency Department Communication in an Academic Medical Center
}

\author{
Abstract \\ Study objective: We characterize communication in an urban, academic medical center emergency \\ department (ED) with regard to the timing and nature of the medical history survey and physical \\ examination and discharge instructions.
}

Methods: Audiotaping and coding of 93 ED encounters (62 medical history surveys and physical examinations, 31 discharges) with a convenience sample of 24 emergency medicine residents, 8 nurses, and 93 nonemergency adult patients.

Results: Patients were $68 \%$ women and $84 \%$ black, with a mean age of 45 years. Emergency medicine providers were $70 \%$ men and $80 \%$ white. Of 62 medical history surveys and physical examinations, time spent on the introduction and medical history survey and physical examination averaged 7 minutes 31 seconds (range 1 to 20 minutes). Emergency medicine residents introduced themselves in only two thirds of encounters, rarely (8\%) indicating their training status. Despite physician tendency (63\%) to start with an open-ended question, only $20 \%$ of patients completed their presenting complaint without interruption. Average time to interruption (usually a closed question) was 12 seconds. Discharge instructions averaged 76 seconds (range 7 to 202 seconds). Information on diagnosis, expected course of illness, self-care, use of medications, time-specified follow-up, and symptoms that should prompt return to the ED were each discussed less than $65 \%$ of the time. Only $16 \%$ of patients were asked whether they had questions, and there were no instances in which the provider confirmed patient understanding of the information.

Conclusion: Academic EDs present unique challenges to effective communication. In our study, the physician-patient encounter was brief and lacking in important health information. Provision of patientcentered care in academic EDs will require more provider education and significant system support.

\section{Comments}

Postprint version. Published in Annals of Emergency Medicine, Volume 44, Issue 3, September 2004, pages 262-267.

Publisher URL: http://dx.doi.org/10.1016/j.annemergmed.2004.02.035

At the time of publication, author Karin V. Rhodes was affiliated with the University of Chicago. Currently, she is a faculty member of the School of Social Policy and Practice at the University of Pennsylvania.

\section{Author(s)}

Karin V. Rhodes, Teri Vieth, Theresa He, Annette Miller, David S. Howes, Olivia Bailey, James Walter, Richard Frankel, and Wendy Levinson 


\section{Resuscitating the Physician-Patient Relationship: Emergency Department Communication in an Academic Medical Center}

Karin V. Rhodes, MD

Teri Vieth, BA, MBA

Theresa He, BA

Annette Miller, RN

David S. Howes, MD

Olivia Bailey, BS

James Walter, MD

Richard Frankel, PhD

Wendy Levinson, MD

From the Health Services Re search Group (Rhodes), Section of Emergency Medicine (Miller, Howes, Walter), University of Chicago, Chicago, IL; the University of Chicago/Pritzker School of Medicine (Vieth, He, Bailey), Chicago, IL; the Indi ana University School of Med icine (Frankel), Indianapolis, IN; The Regenstrief Institute (Frankel), Indianapolis, IN; and the Department of Medicine (Levinson), University of Toronto, Toronto, Ontario, Canada.
Study objective: We characterize communication in an urban, academic medical center emergency department (ED) with regard to the timing and nature of the medical history survey and physical examination and discharge instructions.

Methods: Audiotaping and coding of 93 ED encounters (62 medical history surveys and physical examinations, 31 discharges) with a convenience sample of 24 emergency medicine residents, 8 nurses, and 93 nonemergency adult patients.

Results: Patients were $68 \%$ women and $84 \%$ black, with a mean age of 45 years. Emergency medicine providers were $70 \%$ men and $80 \%$ white. Of 62 medical history surveys and physical examinations, time spent on the introduction and medical history survey and physical examination averaged 7 minutes 31 seconds (range 1 to 20 minutes). Emergency medicine residents introduced themselves in only two thirds of encounters, rarely $(8 \%)$ indicating their training status. Despite physician tendency $(63 \%)$ to start with an open ended question, only $20 \%$ of patients completed their presenting complaint without interruption. Average time to interruption (usually a closed question) was 12 seconds. Discharge instructions averaged 76 seconds (range 7 to 202 seconds). Information on diagnosis, expected course of illness, self care, use of medications, time specified follow up, and symptoms that should prompt return to the ED were each discussed less than $65 \%$ of the time. Only $16 \%$ of patients were asked whether they had questions, and there were no instances in which the provider confirmed patient understanding of the information.

Conclusion: Academic EDs present unique challenges to effective communication. In our study, the physician patient encounter was brief and lacking in important health information. Provision of patient centered care in academic EDs will require more provider education and significant system support. 


\section{Editor's Capsule Summary}

What is already known on this topic

The emergency department (ED) presents unique challenges to effective provider patient communication, such as lack of privacy, noise, frequent interruptions, and lack of an established re lationship.

What question this study addressed

This small pilot study attempts to characterize communication in an urban academic medical center ED with regard to the timing and nature of the history and physical examination and discharge instructions.

What this study adds to our knowledge

Ninety three audiotaped verbal communications between pro vider and patient were examined for rapport building, informa tion gathering, and information giving skills. Patients were frequently interrupted, were given diagnosis and follow up information less than $65 \%$ of the time, and were never asked if they understood the information.

How this might change clinical practice

Communicating effectively with patients is important, and there is much room for improvement. In particular, follow up informa tion is frequently not provided, and patients have little opportunity to seek clarification.

\section{INTRODUCTION}

Effective communication is an essential component of "patient-centered care," 1 of the 6 areas targeted for quality improvement by the Institute of Medicine in their analysis of the US health care system. With lack of privacy, noise, frequent interruptions, expectation of rapid patient turnover, long waiting times, physical factors, and lack of an established relationship, the emergency department (ED) setting presents unique challenges to effective communication. However, ED literature suggests that skillful emergency physicians can establish rapport and trust in the first few minutes of an encounter while rapidly gathering information vital to diagnosis and treatment. ${ }^{1}$ Emergency physicians are not the only providers facing the challenge of maximizing meaningful communication during a short problem-focused visit. The commonality of communication problems and safety issues inherent in busy patient-care settings highlights the importance of research in the area of physician-patient communication. The goal for this project was to characterize communication during ED visits.

A broad range of studies based on coding of audio- and videotape recordings of physician-patient communication have been published in mainstream medical literature. In comparison with other methodologies such as questionnaires, retrospective recall, and interviews, studies based on audio- and videotaping have the advantages of being accurate, reproducible, and relatively easy to code. ${ }^{2}$ As a result, several reliable schemes for coding physicianpatient communication have been developed and compared. ${ }^{3,4}$ Because of limited research on face-to-face communication in the ED setting, we chose methods that would generate new hypotheses while testing existing ones, an approach recommended by Inui et $\mathrm{al}^{3}$ and other health services researchers who have studied physicianpatient relationships. ${ }^{5,6}$

\section{MATERIALS AND METHODS}

\section{Study Design}

This is a descriptive study of audiotaped verbal communication between emergency medicine providers and patients in an inner-city academic medical center ED, which took place during July and August 2000 and 2001. The medical student research assistants were given a structured review of physician-patient communication literature applicable to emergency medicine and educational sessions on research methodology and audiotape analysis.

\section{Selection of Participants}

Study participants were a convenience sample of nonemergency English-speaking patients older than 18 years and presenting to our ED during weekday data collection periods, and the ED providers caring for them. Patients were excluded if they required immediate medical intervention or were cognitively impaired. After obtaining verbal consent from the resident, nurse, and patient, the research assistant placed a small recorder on the bedside table. Recording commenced when the treating physician entered the room.

\section{Data Collection and Processing}

To generate data from audiotapes, a coding scheme was developed in an iterative fashion after the first 20 tapes were listened to, a well-accepted qualitative research technique. ${ }^{7}$ Rapport-building was assessed by analysis of the physician introduction for the presence of a greeting, provider name, and indication of training status. We evaluated the physician's initial solicitation of the patient's chief complaint, coding it as either an open-ended or closed question and noted whether the patient's response was completed (ie, whether the patient finished expressing his or her concern before being interrupted). If the patient was interrupted, we noted the time and nature of the interruption and whether the patient eventually returned to finish explaining the chief complaint. 
Data on information-gathering included overall time to complete the medical history survey and physical examination; the number of external interruptions; whether the history and physical examination were done simultaneously; whether provider questioning was facilitative or interruptive; and whether the physician provided any "orientation" (information about what could be expected during or after the ED visit), health education, or behavioral risk-factor counseling. Coders also noted additional solicitations by the physician that gave the patient an opportunity to elaborate on the initial concern or express additional concerns. All interviews were double coded, and stopwatches were used to measure timerelated variables. Intracoder agreement was more than $80 \%$ on all variables. The primary author independently coded $42 \%$ of the encounters to monitor coding quality and to resolve any interrater discrepancies.

Discharge is the traditional phase of the ED encounter for information-giving. A second research team conducted the discharge portion of this study on a separate sample of patients and providers. The research assistant noted who gave discharge instructions (emergency medicine resident, nurse, or both) and measured the provider-patient interaction time. An experienced emergency physician and nurse coded the discharges for desired elements of information-giving identified in the ED literature. We noted whether the provider asked if the patient had any questions or confirmed that discharge instructions were understood.

\section{Primary Data Analysis}

Data were imported into Stata7 (Stata Corporation, College Station, TX) software and analyzed with descriptive methods. The institutional review board approved the study.

\section{RESULTS}

A total of 166 nonemergency adult patients were approached for the study, and 115 (69\%) agreed to participate. Of these, 93 (81\%) were successfully recorded. Table 1 presents the demographics and characteristics of recorded patients. Patients who did not consent or were not recorded were similar in age, sex, race, and triage severity from those who were recorded. The 24 emergency medicine residents were $70 \%$ men, $10 \%$ black, $80 \%$ white, and 10\% Asian, and 80\% were postgraduate year 2 or above. Of 62 medical history surveys and physical examinations, 2 lacked the quality necessary for coding more than time variables. The average length of the medical history survey and physical examination was 7.5 minutes (range 1 to 20 minutes). Approximately half the medical history surveys and physical examinations experienced external interruptions sufficient to disrupt the initial evaluation. In $52 \%(n=31)$, it was obvious that the provider was simultaneously doing the physical examination while taking the medical history. During the introduction, providers greeted patients $62 \%(n=37)$ of the time and introduced themselves by name $65 \%(n=39)$ of the time. However, they rarely $(n=5,8 \%)$ indicated their status as residents in training. Table 2 presents characteristics of the initial solicitation of chief complaint. The majority of interviews began with an open-ended question, but few patients were allowed to complete their response, with the average interruption occurring in 12 seconds. Nonetheless, coders rated the provider's question style as "facilitating" $68 \%$ of the time (intracoder agreement $83 \%$ ). After the initial evaluation, physicians usually $(\mathrm{n}=53,88 \%)$ provided some orientation about tests or procedures to expect during the ED visit, and half $(n=30)$ of the physicians provided health education or riskbehavior counseling during or after the medical history survey and physical examination. A full graphic representation of each medical history survey and physical examination is shown in Appendix El (available at http:// www.mosby.com/AnnEmergMed). There were no important differences in the length of the medical history survey and physical examination, regardless of whether the initial solicitation was open or closed ( 7.4 versus 7.2

\section{Table 1.}

Patient demographic characteristics.

\begin{tabular}{lc}
\hline Characteristic & $\%$ (No.) \\
\hline Recorded/consenting* & \\
Age, y, mean (SD) & $81(93 / 115)$ \\
Sex & $45(18)$ \\
Male & \\
Female & $32(30)$ \\
Race & $68(63)$ \\
Black & \\
White & $84(78)$ \\
Hispanic & $13(12)$ \\
Triage severity & $3(3)$ \\
Nonurgent & \\
Intermediate & $41(38)$ \\
Urgent & $53(50)$ \\
Disposition & $5(5)$ \\
Discharged & \\
Admitted & $88(82)$ \\
\end{tabular}

*Of 166 approached patients, $115(69 \%)$ consented, but only 93 were successfully recorded. Patients who did not consent (or consented but were not recorded) did not differ significantly in age, sex, race, or triage severity. 
minutes), the patients eventually completed their chief complaints ( 7.6 versus 7.7 minutes), or the physician's style was "facilitating" versus "interrupting" ( 7.4 versus 7.7 minutes). These results were not skewed by specific physician style because individual doctors did not always show consistent interview patterns.

Table 3 presents the discharge instructions for 31 patients. The distribution of the ED staff providing the discharge instructions was equally split between the physicians and nurses, with a small portion of patients receiving instruction from both. During discharge instructions, providers talked an average of 76 seconds (range 7 to 202 seconds), whereas patients talked an average of 14 seconds (range 0 to 75 seconds). Information on diagnosis, expected course of illness, self-care, use of medications, time-specified follow-up, and symptoms that should prompt return to the ED were each discussed less than $65 \%$ of the time. Only $16 \%$ of patients were asked whether they had questions, and there were no instances in which the provider confirmed patient understanding of the information.

\section{Table 2.}

Solicitation of patient's chief complaint $(N=60)$.

\section{Interview Characteristic}

$\%$ (No.)

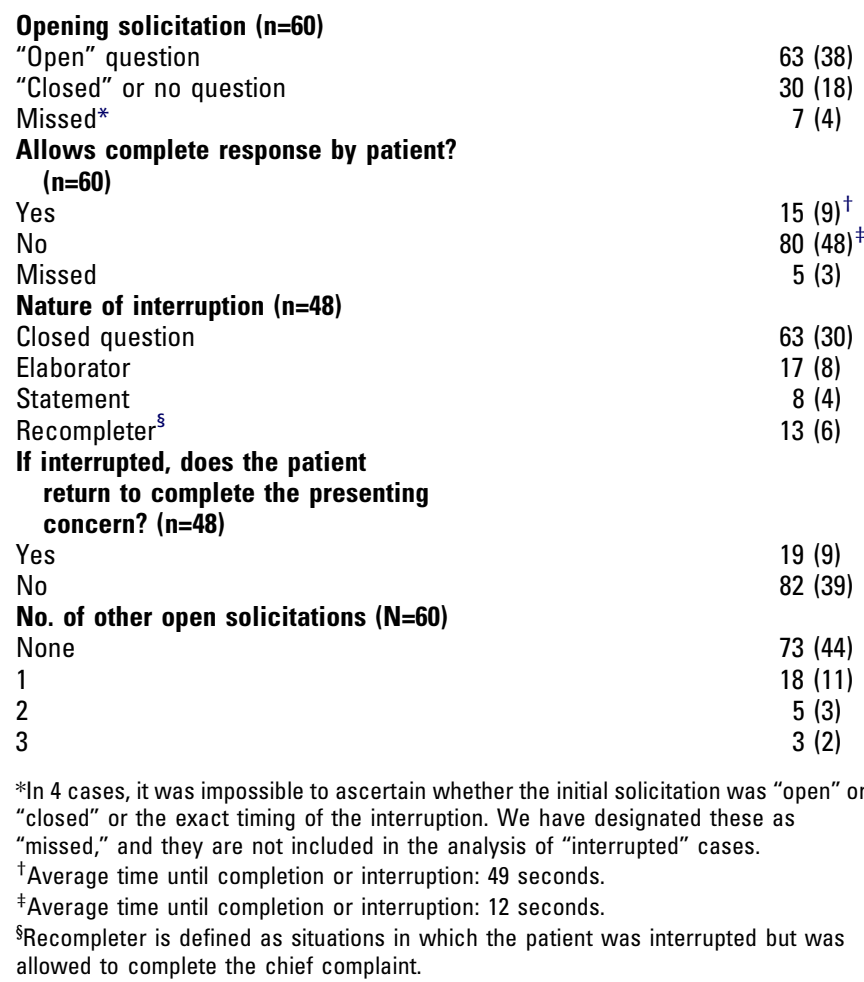

$8(4)$

$13(6)$

$82(39)$

$73(44)$

$18(11)$

$5(3)$

\section{LIMITATIONS}

This was a small pilot study measuring the verbal communication skills of a particular group of emergency medicine residents at one urban academic center. It took place during 2 summers, and the composition of the residents changed during the intervening year. We were unable to compare common omissions or patterns in individual interviews because data were not collected by the physician, limiting us to analyzing by physician descriptors. In addition, our ED has experienced extreme crowding because of increased patient volume and prolonged throughput times, exacerbated by limited inpatient capacity; hence, our experience may not apply to all academic EDs.

Audiotaping does not capture nonverbal elements of communication, which is important, and we coded only verbal discharge instructions. Although the literature supports using complementary written materials, verbal instructions are believed to be more important. Finally, audiotaping may have produced subtle shifts, causing physicians to be "on their best behavior," creating a Hawthorne effect. However, similar studies have demonstrated that the presence of a recording device has minimal impact on provider behavior, especially when time is critical and there is pressure for throughput. ${ }^{2}$

Table 3.

Characteristics of discharge instructions $(N=31)$.

Discharge Component $\%$ (No.)

Who discharged patient

Physician

$45(14)$

Registered nurse

$45(14)$

Both

Patient given a diagnosis

$10(3)$

Prescribed medication(s)

$55(17)$

Verbal instructions about

medications (if prescribed):

Frequency

Dose

44 (12)

Duration of therapy

Possible adverse effects

$26(7)$

None of the above

Some explanation of the expected course of illness

(however minimal)

Some instructions about self-care (however minimal) 48 (15)

Instructed to seek follow-up care 77 (24)

Specified time for follow-up 35 (11)

Instructions about symptoms that should prompt return $65(20)$

to the ED (however minimal)

Patient asked whether he or she had any questions

Patient asked whether he or she understood diagnosis

$16(5)$

or discharge plan 


\section{DISCUSSION}

We found that the emergency medicine resident-patient encounter gave far more attention and time to information-gathering than to information-giving. Emergency medicine residents frequently failed to introduce themselves and rarely indicated their training status. Although the physician initiated the interview with an open question, he or she quickly interrupted the patient, and these patients rarely returned to complete their chief complaint. Information-giving during discharge was generally lacking in content, specific follow-up instructions, and opportunities to ask questions. Of note, "permissive" or "partnership" interview characteristics (ie, open-ended questioning, allowing completion of chief complaint, facilitative style) did not prolong the initial evaluation.

Wissow et $\mathrm{al}^{8}$ used audiotaping in an ED setting and identified that providers with a "patient-centered" style (defined as above a 50\% margin for partnership, interpersonal sensitivity, and information-giving) elicited more patient participation and received higher ratings. Similar studies in primary care have advanced the teaching of communication skills during undergraduate medical training. Perhaps this focus should be extended more widely in the postgraduate years.

The Society for Academic Emergency Medicine's task force on physician-patient communication identified 5 essential tasks of ED communication: establish rapport, gather information, give information, provide comfort, and collaborate; these are skills that are "often telescoped to the point of being almost simultaneous." Although the report states: "Effective communication is not a function of time but rather one of skill," it emphasizes that a few extra seconds spent on each of these tasks is actually time efficient and can decrease inappropriate workup, interpersonal conflict, and litigation and enhance compliance with follow-up care. ${ }^{1}$

The literature supports the concept that rapport is a function of strategy and style. Korsch et $\mathrm{al}^{9}$ found that patient satisfaction was not related to interview length. They detected high levels of dissatisfaction with ED care when parents of asthmatic children expected more concern and sympathy than they received. Less than $25 \%$ of their sample reported being able to discuss the main concern that had prompted the ED visit. Therefore, rather than just measuring rapport as a function of time, we looked at components of rapport such as introduction, greeting, and style of communication and whether the provider allowed complete responses and opportunities for questions and affirmed understanding of discharge instructions. Because rapport must be established within the first moments of the ED encounter, we looked specifically at the opening moments of the interview. The emergency medicine residents' interview techniques are presumably driven by a need to rapidly triage patient complaints. As such, positive changes are likely if emergency medicine providers recognize that time spent allowing patients to complete their chief complaint does not lengthen the interview. In a recent British study, patients completely presented their concerns in an average of 75 seconds, further evidence that uninterrupted patients do not talk extensively. ${ }^{10}$

The discharge period has been identified as a second opportunity to strengthen the therapeutic relationship. Waggoner et al ${ }^{11}$ found that a 5-minute ED exit interview, designed to enhance rapport, increased compliance with follow-up by more than $50 \%$. Reasons to return to the ED and the timing of follow-up care are considered essential components of discharge instructions. Recent work by Sun et $\mathrm{al}^{12}$ found ED patient satisfaction and "willingness to return" to be significantly negatively associated with poor explanation of problem causes. It is notable that only $55 \%$ of patients were told their diagnosis and $60 \%$ were told about symptoms requiring an ED return. Indeed, less than $20 \%$ were informed about the expected course of their illness or injury.

In other high-risk industries (eg, aviation), it is considered essential to understand to whom information is given, ensure that information-giving is completed, and confirm the understanding of critical information. ${ }^{13}$ However, although formal discharge instructions were lacking in content, $50 \%$ of patients received some health information during the medical history survey and physical examination. Nonetheless, half of our ED medical history surveys and physical examinations contained at least 1 external interruption, 35\% of patients were not cautioned about symptoms that should prompt a return, $84 \%$ were not prompted for questions, and patient understanding of the discharge instructions was never confirmed, suggesting a fertile area for improvement of ED safety.

This study suggests that key communication variables associated with rapport, satisfaction, and compliance are somewhat lacking in our provider-patient interactions. Reinforcing the value of quality communication may be particularly important in training environments characterized by frequent interruptions and the need to multitask. Future studies should assess the relationship between effective communication and outcomes of emergency care. 
We wish to acknowledge the University of Chicago Pritzker School of Medicine Student Summer Research Program, the Robert Wood Johnson Clinical Scholars Program, and the emergency medicine residents and faculty at the University of Chicago.

Author contributions: KVR, WL, RF, and JW conceived the study and designed the trial. KVR, WL, and DSH obtained funding. KVR, TH, OB, and $\mathrm{AM}$ were responsible for data collection and database management. KVR, TH, TV were responsible for data analysis. KVR and TH wrote the first draft, and KVR and TV wrote the final version, with input from RF and DSH. All authors reviewed final versions, but KVR takes responsibility for the paper as a whole.

Received for publication March 14, 2003. Revisions received July 15, 2003; September 11, 2003; December 13, 2003; January 29, 2004; and February 18, 2004. Accepted for publication February 23, 2004.

Available online July 17, 2004.

Presented as an abstract at the Society for Academic Emergency Medicine annual meeting, Atlanta, GA, May 2001.

Funded in the preliminary phases of a randomized controlled trial by the Agency for Health Care Research and Quality (AHRO HS11096-02).

Dr. Rhodes is supported by a career development award from the National Institute of Mental Health (K23/PA-00-004).

Address for reprints: Karin V. Rhodes, MD, Section of Emergency Medicine, The University of Chicago, 5841 South Maryland Avenue, MC 5068, Room L545, Chicago, IL 60637; 773-834-7467, fax 773-702-

3135; E-mail krhodes@medicine.bsd.uchicago.edu.

\section{REFERENCES}

1. Knopp R, Rosenzweig S, Bernstein E, et al. Physician patient communication in the emergency department, part 1. Acad Emerg Med. 1996;3:1065 1069.

2. Jordan B, Henderson A. Interaction analysis: foundations and practice. J Learn Sci. 1995;4:39 103.

3. Inui TS, Carter WB, Kukull WA, et al. Outcome based doctor patient interaction analysis, I: comparisons of techniques. Med Care. 1982;20:535 549.

4. Frankel RM. Cracking the code: theory and method in clinical communication analysis. Health Commun. 2001;13:101 110.

5. Inui TS, Carter WB. Problems and prospects for health services research on provider patient communication. Med Care. 1985;23:521 538.

6. Simpson MR, Buckman R, Stewart M, et al. Doctor patient communication: the Toronto consensus statement. BMJ. 1991;303:1385 1387.

7. Crabtree BF, Miller WL, eds. Doing Qualitative Research. 2nd ed. Thousand Oaks, CA: Sage Publications; 1999.

8. Wissow LS, Roter D, Bauman LJ, et al, for the National Cooperative Inner city Asthma Study. Patient provider communication during the emergency department care of children with asthma. Med Care. 1998;36:1439 1450.

9. Korsch BM, Gozzi EK, Francis V. Gaps in doctor patient communication: doctor patient satisfaction. Pediatrics. 1968;42:855 871.

10. Langewitz W, Denz M, Keller A, et al. Spontaneous talking time at start of consultation in outpatient clinic: cohort study. BMJ. 2002;325:682 683.

11. Waggoner DM, Jackson EB, Kern DE. Physician influence on patient compliance: a clinical trial. Ann Emerg Med. 1981;10:692 696.

12. Sun BC, Adams J, Orav EA, et al. Determinants of patient satisfaction and willingness to return with emergency care. Ann Emerg Med. 2000;35:426 434. 13. Frankel RM. "Captain, I was trying earlier to tell you that you made a mistake": deference and demeanor at 30,000 feet. In: Peyton JK, Griffin P, Wolfram W, et al, eds. Language in Action: New Studies of Language in Society. Kresskill, NJ: Hampton Press; 2000:289 299 . 